\title{
FINITE RANK TORSION-FREE ABELIAN GROUPS UNISERIAL OVER THEIR ENDOMORPHISM RINGS
}

\author{
JUTTA HAUSEN ${ }^{1}$
}

\begin{abstract}
An abelian group is $E$-uniserial if its lattice of fully invariant subgroups is totally ordered. Finite rank torsion-free reduced $E$-uniserial groups are characterized. Such a group is a free module over the center $C$ of its endomorphism ring, and $C$ is a strongly indecomposable discrete valuation ring. Properties similar to those of strongly homogeneous groups are derived.
\end{abstract}

The results. In the sequel all groups will be abelian. This paper was motivated by the recent study of additive groups of valuation rings (i.e. rings whose lattice of two-sided ideals forms a chain) $[3,4,6]$. Obviously, the additive group of a valuation ring is uniserial regarded as a module over its endomorphism ring. We call such groups $E$-uniserial for short. In [5] we characterized the $E$-uniserial groups up to torsion-free reduced direct summands. Combining the results of [5] with Theorem 1 below yields the structure of all $E$-uniserial groups of finite torsion-free rank.

We shall prove

THEOREM 1. Let $G$ be a torsion-free reduced abelian group of finite rank. Then the following conditions are equivalent.

(a) $G$ is E-uniserial.

(b) $G \simeq H^{m}$ where $H$ is a strongly indecomposable E-uniserial group.

(c) $G$ is a free module over a valuation ring.

(d) The center $C=\operatorname{Cent} E(G)$ of the endomorphism ring of $G$ is a strongly indecomposable discrete valuation $E$-ring, and $G$ is a free $C$-module.

(e) $E(G) \simeq \operatorname{Mat}_{m}(C)$ where $C$ is a discrete valuation ring and rank $G=m$. (rank $C$ ).

We use the phrase "discrete valuation ring" in the sense of Kaplansky [7, p. 42]. In particular, a discrete valuation ring is a principal ideal domain. A (discrete) valuation $E$-ring is a (discrete) vaiuation ring which is also an $E$-ring. $E$-rings were introduced by $\mathrm{P}$. Schultz [10] as the rings whose additive endomorphisms are given by left multiplication with elements. Throughout, $E(G)$ is the endomorphism ring of $G$, the center of a ring $R$ is denoted by Cent $R$, and $\operatorname{Mat}_{m}(R)$ is the ring of $m \times m$ matrices with entries in $R$.

Received by the editors February 16, 1984.

1980 Mathematics Subject Classification. Primary 20K15.

Key words and phrases. E-uniserial, torsion-free abelian group, valuation domain.

${ }^{1}$ This research was supported in part by a University of Houston-University Park Research Enabling Grant. 
Torsion-free $E$-uniserial groups are $p$-local and strongly irreducible. Reid calls a torsion-free abelian group $G$ strongly irreducible (irreducible) if, for every fully invariant subgroup $S \neq 0$ of $G$, the quotient group $G / S$ is bounded (torsion) [8, 9]. Arnold's strongly homogeneous groups are irreducible [1]. The group $G$ is strongly homogeneous if the automorphism group of $G$ acts transitively on the set of pure. rank-one subgroups of $G$. Given a torsion-free $p$-local strongly homogeneous group $G$, it is easy to see that every proper fully invariant subgroup of $G$ is of the form $p^{n} G$ for some natural number $n$. Thus, the $E$-uniserial torsion-free groups are sandwiched between the local strongly irreducible groups and the local strongly homogeneous groups. We will show that both containments are proper. The torsion-free $E$-uniserial groups share a number of properties with Arnold's strongly homogeneous groups [1]:

COROLlARY 2. Let $G$ be a finite rank torsion-free reduced E-uniserial group. Then:

(1) $G$ is indecomposable if and only if $G$ is strongly indecomposable.

(2) If $G$ is strongly indecomposable then $G \simeq[E(G)]^{+}$and $E(G)$ is a discrete valuation $E$-ring.

(3) If $G \simeq H^{m}$, with $H$ strongly indecomposable, and if $A$ is a direct summand of $G$, then $A \simeq H^{n}$ for some $n \leqslant m$.

(4) If $B$ is another torsion-free reduced E-uniserial group then $G \simeq B$ if and only if rank $G=\operatorname{rank} B$ and Cent $E(G) \simeq$ Cent $E(B)$.

A result of Bowshell and Schultz states that if the additive groups of two torsion-free unital rings are quasi-isomorphic and one of them is an $E$-ring, then so is the other [2, p. 210, 3.9 (i)]. By the above results, a finite rank torsion-free strongly indecomposable group $H$ is $E$-uniserial if and only if $H$ is the additive group of a (discrete) valuation $(E$-) ring. We will give an example of two quasi-isomorphic strongly indecomposable $E$-rings of finite rank, one of which is a valuation ring and the other is not. However, we have the following result:

Proposition 3. Let $R$ and $S$ be two unital valuation E-rings whose additive groups are torsion-free and strongly indecomposable of finite rank. If $R^{+} \dot{\sim} S^{+}$, then $R \simeq S$ as rings.

This is used to prove that the $E$-uniserial groups share another property of Arnold's strongly homogeneous groups [1]:

Proposition 4. Two torsion-free reduced E-uniserial groups of finite rank are quasi-isomorphic if and only if they are isomorphic.

The proofs. Let $G$ be a torsion-free reduced abelian group of finite rank. The following results will be helpful. They are easy to prove [5, 9]. Throughout, $C=$ Cent $E(G)$ denotes the center of the endomorphism ring of $G$.

LeMma 5. (i) Let $G$ be E-uniserial. Then $G$ is p-local for some prime $p, G$ is strongly irreducible, and $G$ is $E$-cyclic.

(ii) Let $G \simeq H^{m}$. Then $G$ is E-uniserial if and only if $H$ is E-uniserial, and $G$ is strongly irreducible if and only if $H$ is strongly irreducible.

(iii) If $G$ is irreducible then $G$ is a torsion-free $C$-module. 
LEMMA 6. Let $T$ be a ring with additive group torsion-free of finite rank and let $R$ and $S$ be subrings of $T$ such that $R^{+} \doteq S^{+}$. Then

(i) Cent $R \doteq$ Cent $S$, and

(ii) if $R$ is finitely generated as a module over Cent $R$ then $S$ is finitely generated as a module over Cent $S$.

Proof. By hypothesis, $n R \subseteq S$ and $n S \subseteq R$ for some positive integer $n$. One verifies that then $n$ Cent $R \subseteq$ Cent $S$ and $n$ Cent $S \subseteq$ Cent $R$, proving (i). For (ii) observe that, if $\left\{r_{i}\right\}$ generates $R$ as a module over Cent $R$, the (Cent $S$ )-module generated by $\left\{n r_{i}\right\}$ contains $n^{3} S$ and, thus, has finite index in $S$.

Of central importance will be the following result. We let $E=E(G)$. Again, $C=$ Cent $E$.

LEMMA 7. Let $G$ be a torsion-free $C$-module. Then $C$ is a domain, and there exists an order isomorphism from the poset of principal ideals of $C$ into the lattice of E-submodules of $G$.

Proof. Consider $G$ embedded into the finite-dimensional vector space $V=Q \otimes G$. Then $E$ consists of all linear transformations of $V$ mapping $G$ into $G$. Note that every $0 \neq \zeta \in C$ has an inverse in $\operatorname{Hom}_{Q}(V, V)$ so that $C$ is a domain. Let $\zeta \in C$ and associate with the principal ideal $C \zeta$ the $E$-submodule $G \zeta$. This is a well-defined monotone map. If $\zeta_{i} \in C$ such that $G \zeta_{1} \subseteq G \zeta_{2}$ then $G \zeta_{1} \zeta_{2}^{-1} \subseteq G$ so $\zeta_{1} \zeta_{2}^{-1}$ is in $E$ and, hence, in $C$. Consequently, $C \zeta_{1} \subseteq C \zeta_{2}$.

We are ready for the

Proof of THEOREM 1. The additive group of a valuation ring is $E$-uniserial. Thus, by Lemma 5(ii), (a) follows from each of (b) and (c), and (d) implies (c). Assume (a). In order to derive (d) we first show that $C$ is a discrete valuation ring. By Lemma 5 , $G$ is strongly irreducible and, thus, a torsion-free $C$-module. Lemma 7 implies that $C$ is a domain whose principle ideals are totally ordered. Since $C$ is reduced it follows that every nonzero (principal) ideal of $C$ contains some $p^{n} C$ and, consequently, has finite index in $C$. Hence $C$ is Noetherian, thus, a principal ideal domain, and a discrete valuation ring. By J. D. Reid's fundamental theorem [8, 5.5, p. 59], the strong irreducibility of $G$ implies $G \doteq H^{m}$ with $H$ strongly indecomposable and strongly irreducible, using Lemma 5(ii) and the fact that strong irreducibility is invariant under quasi-isomorphism. But then $H \doteq R^{+}$where $R$ is a strongly indecomposable $E$-ring [9, p. 49, Theorem 6]. Hence $G \dot{\sim} H^{m} \doteq\left(R^{+}\right)^{m}$, so

$$
C \doteq \operatorname{Cent}_{m}(R) \simeq R,
$$

and $E(G)$ is finitely generated over $C$, by Lemma 6 . Finitely generated torsion-free modules over principal ideal domains are free. By [2, 3.9 (i), p. 210], $C \doteq R$ is an $E$-ring, and we have derived (d) from (a). Obviously, (e) follows from (d). The proof will be completed once we show that (e) implies (b). Assume (e), fix an isomorphism between $E(G)$ and $\mathrm{Mat}_{m}(C)$, and let $\varepsilon_{i}$ be the endomorphism of $G$ corresponding to the diagonal matrix $E_{i}$ with 1 in $(i, i)$-position and zeros elsewhere. Then $G=G \varepsilon_{1}$ $\oplus \cdots \oplus G \varepsilon_{m}, E\left(G \varepsilon_{i}\right) \simeq C$, and $G \varepsilon_{i} \simeq G \varepsilon_{j}$ for all $i$ and $j$. Let $H \simeq G \varepsilon_{i}$. Then $G \simeq H^{m}, E(H) \simeq C$, and $\operatorname{rank} H=\operatorname{rank} C$. Let $E=E(H)$ and let $S$ and $T$ be two 
$E$-submodules of $H$. Assume, by way of contradiction, that $S \nsubseteq T$ and $T \nsubseteq S$. Then there exist $s \in S$ and $t \in T$ such that $s \notin T$ and $t \notin S$. Since $E \simeq C$ is a discrete valuation ring and $H$ is a torsion-free $E$-module, every finitely generated $E$-submodule of $H$ is free and therefore cyclic. Hence $s E+t E=x E \simeq E$ for some $x \in H$ and, since the submodules of $E$ are totally ordered, $s E \subseteq t E$ or $t E \subseteq s E$. Thus, either $s \in T$ or $t \in S$, a contradiction.

Proof of Corollary 2. Part (b) of Theorem 1 implies (1), part (d) implies (2) and (4). For (3) observe that $A$ is a $C$-submodule of the free $C$-module $G$, that submodules of free modules over principal ideal domains are free, and that

$$
C=\operatorname{Cent} E(G) \simeq \operatorname{Cent} E(H)=E(H), \quad E(H)^{+} \simeq H .
$$

Proof of Proposition 3. By (2) of Corollary 2, both $R$ and $S$ are discrete valuation $E$-rings. Let $x R$ be the unique maximal ideal of $R$. Then $x R$ contains a unique rational prime $p$ and $p R=x^{t} R$ for some positive integer $t$. The hypothesis implies $R \subseteq G \subseteq K$ where $G \simeq S^{+}, p^{n} G \subseteq R$ for some $n$, and $K$ is the quotient field of $R$. Let $\varepsilon \in E(G)$. Since $p^{n} E(G) \subseteq E\left(R^{+}\right)$and $R$ is an $E$-ring, $p^{n} \varepsilon=r \cdot 1_{R}$ for some $r \in R$ and hence $\varepsilon=\left(p^{-n} r\right) \cdot 1_{K}$, using the fact that $K=Q \otimes R[2,3.14, \mathrm{p}$. 211]. It follows that, for every positive integer $k, \varepsilon^{k}=\left(p^{-k n} r^{k}\right) \cdot 1_{K}$. But then $p^{n}\left(p^{-k n} r^{k}\right) \in R$ for each $k$. Let $m$ be such that $r R=x^{m} R$. Then $x^{t n-t k n+m k} \in R$, which implies $t n \geqslant k(t n-m)$ for each $k$ and thus $t n \leqslant m$. Hence, $r \in p^{n} R$ and $\varepsilon=s \cdot 1_{K}$ for some $s \in R$. This being true for all $\varepsilon$ in $E(G)$ shows that $R$ is an $E(G)$-submodule of $G$. By (2) of Corollary $2, R^{+} \simeq[E(G)]^{+} \simeq S^{+}$and $R \simeq E\left(R^{+}\right)$ $\simeq E\left(S^{+}\right) \simeq S$.

We can now easily complete the

Proof of Proposition 4. Let $A$ and $B$ be two finite rank torsion-free reduced $E$-uniserial groups which are quasi-isomorphic. By part (d) of Theorem 1 and Lemma 6, the centers of their endomorphism rings are discrete valuation $E$-rings whose additive groups are quasi-isomorphic. By Proposition 3 the centers are isomorphic, and $A \simeq B$ since $A$ and $B$ have equal rank.

EXAMPLES. The construction of the following ring $R$ is due to C. Vinsonhaler. Let $p$ be a prime and consider the polynomial $f(X)=X^{5}-X^{3}-p$. One verifies that $f$ is irreducible in $Z[X]$. Let $x$ be a complex root of $f$, let $K=Q(x)$, and let $S=Z[x]$. Then $S / x S \simeq Z(p)$ so $x S$ is a maximal ideal. Let $R=S_{x S}$ be the localization of $S$ at $x S$. Then

(i) $R$ is a discrete valuation ring with maximal ideal $x R$; and

(ii) $p R=x^{3} R$.

Since $x^{n} R / x^{n+1} R \simeq Z(p)$ for every nonnegative integer $n$, the rank of $R / p R$ is three, which implies $R \neq H^{5}$. Since $G$ is $E$-uniserial, (b) of Theorem 1 and (2) of Corollary 2 imply

(iii) $R$ is a strongly indecomposable $E$-ring.

Let $G=R+Z_{(p)} \cdot 1 / p \subseteq K$, where $Z_{(p)}$ denotes the set of all rational numbers with denominators prime to $p$. Then

(iv) $p G \subseteq R \subseteq G \subseteq Q \otimes R=K$,

which implies $p E(G) \subseteq E\left(R^{+}\right)=R \cdot 1_{R}$. One verifies that $E(G)$ consists precisely of 
multiplications with elements of the form $n+p r$, where $n \in Z_{(p)}$ and $r \in R$. Thus $1 / p$ generates $G$ as an $E(G)$-module and

(v) $G$ is $E$-cyclic.

We claim that

(vi) $G$ is not $E$-uniserial.

Assume the contrary. Since $x \notin x^{2} E(G) \subseteq x^{2} R$, we then have $x^{2} E(G) \subseteq x E(G)$. Thus $x^{2}=x(n+p r)$ for some $n \in Z_{(p)}$ and $r \in R$, so $x$ divides $n+p r$. Since $p R=x^{3} R$ if follows that $n \in Z_{(p)} \cap x R=p Z_{(p)}$. But then $p$ divides $x^{2}$, which is a contradiction.

The ring $R$ and the group $G$ constructed above show the following:

(1) Not every $E$-uniserial group is strongly homogeneous.

In fact, since $R \subsetneq x R \subsetneq p R$, not every proper fully invariant subgroup of $R^{+}$is of the form $p^{n} R^{+}$so that $R^{+}$is $E$-uniserial but not strongly homogeneous. (A different argument would have been the observation that not every element in $R$ is an integral multiple of a unit [1, Theorem 1, p. 67].)

(2) Not every (strongly indecomposable $E$-cyclic) strongly irreducible $p$-local group is $E$-uniserial.

The group $G$ above provides an example. Since $G \doteq R^{+}, G$ is strongly indecomposable.

(3) An $E$-ring whose additive group is quasi-isomorphic to the additive group of a valuation $E$-ring need not be a valuation ring.

The example here is $S=E(G)$ which is quasi-isomorphic to $E\left(R^{+}\right) \simeq R$, but $S$ is not a valuation ring since $G$ is not $E$-uniserial (Theorem $1(\mathrm{e})$ ).

\section{REFERENCES}

1. D. M. Arnold, Strongly homogeneous torsion free abelian groups of finite rank, Proc. Amer. Math. Soc. 56 (1976), 67-72.

2. R. A. Bowshell and P. Schultz, Unital rings whose additive endomorphisms commute, Math. Ann. 228 (1977), 197-214.

3. S. Feigelstock, The additive groups of rings with totally ordered lattice of ideals, Quaestiones Math. 4 (1981), 331-335.

4. __ Additive groups of rings, Research Notes in Math., vol. 83, Pitman, Boston, Mass., 1983.

5. J. Hausen, Abelian groups which are uniserial as modules over their endomorphism rings, Abelian Group Theory, Lecture Notes in Math., vol. 1006, Springer-Verlag, Berlin and New York, pp. 204-208.

6. The additive group of rings with totally ordered ideal lattices, Quaestiones Math. 6 (1983), $323-332$.

7. I. Kaplansky, Infinite abelian groups, rev. ed., Univ. of Michigan Press, Ann Arbor, 1969.

8. J. D. Reid, On the ring of quasi-endomorphisms of a torsion-free group, Topics in Abelian Groups, Scott, Foresman, Chicago, 1963, pp. 51-68.

9. __ Abelian groups finitely generated over their endomorphism rings, Abelian Group Theory, Lecture Notes in Math., vol. 874, Springer-Verlag, Berlin and New York, 1981, pp. 41-52.

10. P. Schultz, The endomorphism ring of the additive group of a ring, J. Austral. Math. Soc. 15 (1973), 60-69.

Department of Mathematics, University of Houston - University Park, Houston, TeXas 77004 